litischen Offiziere gedient. Indem er diese Äußerungen mit großer Sorgfalt und Sachkenntnis zusammenstellte, hat er einen wesentlichen Beitrag zur Klärung der über zwei Dutzend Militärputsche und -Regime und damit zur Zeitgeschichte geliefert.

Nach einem umfangreichen Kapitel über die Militärrevolten und -Herrschaften in den einzelnen Ländern hat der Verfasser in weiteren Kapiteln diese Regime klassifiziert, die Verwurzelung der Militärherrschaft in der arabischen Vergangenheit und im Islam skizziert, das Offizierskorps der einzelnen Länder charakterisiert, die Motive der Militärpolitiker untersucht, die Zusammenhänge zum Sozialismus verfolgt und schließlich seine Schlußfolgerungen gezogen. Anhänge bringen Übersichten über die soziale Herkunft der ägyptischen Offiziere, über die Ränge in den arabischen Armeen sowie über das wichtigste einschlägige Schrifttum.

Wenn der Verfasser in seiner Zusammenfassung auch keineswegs zu der Folgerung gelangen kann, daß den arabischen Militärregimen, oder auch nur den wichtigsten von ihnen, einheitliche Motive zugrunde liegen, so zitiert er doch eine Äußerung von Halpern (S. 464), daß sich die Armee aus einer Prätorianergarde $\mathrm{zu}$ einem Vortrupp gewandelt habe; sie betrachte sich als Verkörperung der neuen Mittelklasse und glaube sich dazu bestimmt, die Revolution zum Siege zu führen. Falls dieser Anspruch den Gegebenheiten entspräche, so könnte man vielleicht die Militärregime als ausgleichende und stabilisierende Elemente betrachten, die den Fortschritt junger Staaten verbürgen, solange zivile Organe wie Parteien und Parlamente noch nicht funktionieren. Das vorliegende Werk bestätigt eine solche Annahme indessen nicht, zeigt vielmehr, daß persönlicher Egoismus bei den Militärpolitikern durchaus überwiegt.

Trotzdem ist angesichts der Bemühungen der jungen Staaten, aus der Enge und Starre überkommener Traditionen herauszufinden und den Anschluß an das moderne Leben zu finden, das Ein- greifen und der temporäre Einsatz des einzigen organisierten Machtapparats in das noch unausgewogene staatliche Leben fast unvermeidlich.

Der Verfasser schließt seine Schlußfolgerungen mit dem Satz: „Pluralismus und Militärdiktatur widersprechen einander. Der angebliche "natürliche Kurs" einer Beherrschung des politischen, sozialen und geistigen Lebens der arabischen Völker durch das Offizierskorps führt in eine Sackgasse. Nicht die Militärpolitiker werden ihre Völker in einem Geist der Toleranz dahin führen, daß sie ihr Potential im Sinne der großen Zukunft einsetzen, die sie erwartet". (S. 481). Dem muß jedoch die Frage entgegengehalten werden, ob die arabischen Verhältnisse heute und auf absehbare Zeit einen solchen Pluralismus zulassen oder ob sich nicht in Arabien nur eine autoritäre Militärdiktatur oder eine Einparteienherrschaft als Alternative zu den patriarchalischen Monarchien anbietet.

Conrad Oehlrich

\section{Franz Schurmann}

Ideology and Organization in

\section{Communist China}

2nd enlarged edition. University of California Press,

Berkely and Los Angeles 1968.

LII, 642 S. US $\$ 4,95$ (broschiert).

Ping-Ti Ho and TAng Tsou, editors

China in Crisis

Vol. 1: China's Heritage and the Communist Political System.

The University of Chicago Press,

Chicago 1968.

XIV, 803 S. (2 Halbbände). US $\$ 20$,-

PING-Ti Ho, editor

China in Crisis

Vol. 2: China's Policies in Asia and America's Alternatives.

The University of Chicago Press, Chicago 1968. IX, 484 S., US $\$ 10$,-

Das seit Erscheinen der ersten Auflage im Jahre 1966 als eine der besten Arbeiten über die Volksrepublik China bekannte Werk Schurmanns legt man als 
deutscher Leser mit einem Gefühl der Bedrückung aus der Hand: Wann wird die deutsche Chinaforschung wieder so weit sein, derartigen Untersuchungen etwas Gleichwertiges zur Seite zu stellen? Die Arbeit Schurmanns ist aus langjähriger intensiver Beschäftigung mit dem Material und aus der Diskussion mit den Kollegen im Bereiche der an amerikanischen Hochschulen heute fest etablierten gegenwartsbezogenen Chinaforschung entstanden und beweist, daß Analysen oberflächlich bleiben müssen, die man bei uns - aufgescheucht durch Ereignisse wie die Bildung der Volkskommunen oder die Große Proletarische Kulturrevolution - kurzfristig und marktgerecht vor$\operatorname{leg} t^{1}$.

Der eigentlichen organisatorischen Struktur des heutigen China hat Schurmann die vier zentralen Kapitel (Partei; Regierung; Management; Kontrolle) gewidmet. Er stellt uns jedoch kein dürres Gerüst organisatorischer oder administrativer Zusammenhänge hin, sondern erfüllt diese mit dem von der heute in China vorherrschenden Ideologie erfüllten Leben. Und der Darstellung dieser Ideologie widmet er das einleitende Kapitel. Allein schon dieses Kapitel ist eines der bedeutsamsten, das über den chinesischen Kommunismus geschrieben wurde, da es Begriffe definiert und einordnet, die nur allzuoft mißverstanden und falsch angewendet werden. Vor allem das, was außerhalb Chinas als "Maoismus" bekannt ist, findet hier eine seiner Bedeutung entsprechende Erklärung. Die Stellung der „Gedanken Mao Tse-tungs" neben dem Marxismus-Leninismus und ihre Wandlungsfähigkeit, die „praktische“ Ideologie Maos, die Handlungen hervorrufen soll, seine Lehre von den Widersprüchen und seine Auffassung von „Demokratie" sind es, die heute die Ideologie Chinas beherrschen.

Wie diese Ideologie die Organisationen des Staates, der Partei, der Wirtschaft durchdringt, wie hier im einzelnen die
Widersprüche wirken - sei es zwischen "rot" und „sachkundig“, sei es zwischen Demokratie und Zentralismus, sei es zwischen Manager und Kader (dessen Funktion Schurmann in einer kühnen Parallele mit der Funktion des Samurai im Japan des ausgehenden TokugawaShogunats vergleicht) - , bildet den Inhalt der schon erwähnten Zentralkapitel.

Die Wirklichkeitsnähe, die dieses ungewöhnliche Buch geradezu zu einer spannenden Lektüre macht, gipfelt in den beiden Schlußkapiteln über die Städte und die Dörfer Chinas, deren Inhalt die Entwicklung städtischer Volkskommunen und die Umwandlung der Agrargesellschaft von der Bodenreform bis zur Volkskommune bildet.

Diese hier stichwortartig dargestellten Kapitel kannte man im wesentlichen bereits aus der ersten Auflage von 1966. $\mathrm{Da}$ so bald schon eine zweite Auflage erschien, beweist den Eindruck, den das Buch in der Fachwelt hervorgerufen hat. Eine neue Auflage wurde aber auch nötig, weil die 1966 begonnene Kulturrevolution in China weitere Klärungen ermöglicht hat und weil der Autor seinen Kritikern recht gibt, denen in der ersten Auflage die 50er Jahre gegenüber den 60er Jahren zu sehr im Vordergrunde gestanden haben. Schurmann fügt in der 2. Auflage deshalb eine Ergänzung an, in der er die Ereignisse vom "Großen Sprung nach vorn" bis zur "Großen Proletarischen Kulturrevolution" mit seinen früheren Forschungsergebnissen konfrontiert. Er kommt dabei zu Schlußfolgerungen, die im Widerspruch $\mathrm{zu}$ landläufigen Auffassungen stehen, die aber gerade wegen der von ihm geleisteten Vorarbeit überzeugen.

Schurmann vertritt zunächst die Meinung, daß es nicht die pragmatische "Anpassung" gewesen ist, mit der man die Folgen des "Großen Sprungs" überwand und die man im Westen nicht selten mit einer Art Schadenfreude zur Kenntnis genommen hat, die die 60er Jahre kennzeichnete. Das Thema der

$1 \mathrm{Vgl}$. dazu auch die Besprechung des Buches von Giovanni Blumer, „Die Chinesische Kulturrevolution 1965/67“, VURR 1969, S. 241 f. 
60er Jahre waren vielmehr der Neuaufbau der Parteiorganisation sowie die Festigung der Kontrolle und der Parteidisziplin. Nicht soziale oder ökonomische Fragen, sondern ideologische und politische Probleme bestimmen die Diskussion. Damit wird es aber auch möglich, die Kulturrevolution, deren Bedeutung für die gesamte Menschheit Schurmann hervorhebt (S. 521), in die Entwicklung Chinas einzuordnen und den Gegensatz zwischen Mao Tse-tung und Liu Shao-ch'i zu verstehen.

Liu hatte versucht, die Kommunistische Partei, deren Einfluß im Großen Sprung und in den nachfolgenden Jahren zurückgegangen war, wieder zu stärken und sie durch schrittweise Beeinflussung der Zellen der chinesischen Gesellschaft wieder in ganz China fest zu verankern. Liu glaubte, daß revolutionäres Bewußtsein sich nur durch ideologisch und organisatorisch kontrollierte $\mathrm{Ge}$ hirnwäsche aufbauen läßt. Er glaubte, geleitet durch seine pro-sowjetischen Neigungen, an den Zentralismus und an die Herrschaft der Organisation (vgl. S. 515 ff.).

Mao dagegen glaubt an die Massen. Er ist der Überzeugung, daß die Volkskommunen nur überleben können, wenn sie von den Bauern selbst kontrolliert werden. Er wendet sich gegen eine zu rasche Stabilisierung, die nur zur Herrschaft neuer Klassen führen und damit die für die Entwicklung Chinas nötige schöpferische Unruhe abtöten muß. Damit steht Mao Marx, Liu dagegen Lenin näher. Nicht Liu, wie im Westen gemeinhin angenommen wird, sondern Mao hat sich als der Pragmatiker erwiesen. Ein Beweis dafür ist die stabile Lage der Landwirtschaft gerade während der Kulturrevolution, die sicherlich hätte zusammenbrechen müssen, wenn es Liu gelungen wäre, den Einfluß der Partei auf dem Lande durchzusetzen.

Die Kontrolle der Partei über die Volkskommunen hat in der chinesischen Geschichte ihre Parallele in der Kontrolle der Gentry, die sich zwischen Bevölkerung und Staat geschoben hatte und gegen die sich die alte Institution der Bauern-Soldaten richtete, die im Bünd- nis Maos mit der Volksbefreiungsarmee und der Wiederbelebung des Milizsystems neu entstanden ist. Und gerade in diesem Zusammenhang erweist sich die allgemeine Bedeutung des Kampfes zwischen Liu und Mao: Am Beispiel der USA zeigt Schurmann, daß die Entfremdung $z$ wischen dem immer mächtiger werdenden Staat und der Gesellschaft überall zunimmt. Diese Entfremdung in erster Linie ist es, die Mao Tsetung bekämpft. Deshalb hat Schurmann nach Meinung des Rezensenten auch recht, wenn er sagt (S. 505): "China hat sich mit den Problemen der Modernisierung und Industrialisierung in einem weiteren und tieferen Ausmaß auseinandergesetzt als irgendein anderes Land der Welt. Selbst wenn die Chinesen diese Probleme nicht lösen, bleibt China mit allen seinen Erfolgen und Mißerfolgen, mit seinen guten und bösen Seiten, ein Modell, das die Welt gut studieren sollte."

Die zweite Auflage seines Buches, das im übrigen mit einigen Abbildungen, mit ciner Zeittafel (1920-1967) und mit einem chinesischen Glossar ausgestattet ist, findet ihre Ergänzung in einem Beitrag des gleichen Autors zum Thema: "The Attack of the Cultural Revolution on Ideology and Organization", in dem zweiten hier besprochenen Werk, das Beiträge zur Konferenz anläßlich der Gründung des Center for Policy Study der Universität Chicago (1966/ 67) zusammengefaßt. Dieser Beitrag enthält zunächst eine Zusammenfassung des Hauptteils seines Buches und wendet sich dann der Kulturrevolution zu. Jedoch scheint er früher als die oben erwähnte Ergänzung geschrieben worden $\mathrm{zu}$ sein, deren Erkenntnisse enger mit der chinesischen Entwicklung selbst verknüpft sind, als dies im vorliegenden Beitrag der Fall ist. Dennoch ist auch dieser Beitrag lesens- und bedenkenswert, der die Kulturrevolution und die in ihrem Verlaufe erfolgten Säuberungen zu den großen Säuberungen Stalins in den dreißiger Jahren in Parallele setzt.

Der erste, von Ping-ti Ho und Tang Tsou herausgegebene Band enthält 13 
Beiträge Kwang-ching Lius, Albert Feuerwerkers, Martin Wilburs, Benjamin Schwartz', Chalmers Johnsons, John Lewis', C. K. Yangs, Franz Schurmanns, Francis L. K. Hsus, Ta-chung Lius, Alexander Ecksteins, S. N. Eisenstadts und der beiden Herausgeber sowie die doppelte Anzahl von Kommentaren zu den Beiträgen von bekannten Autoren wie Derk Bodde, Herbert Franke, Ezra Vogel, Gungwu Wang u. a. Die Themen dieses Bandes erstrecken sich vom historischen Erbe des heutigen China über die Revolutionsjahre vor Ausbruch des chinesisch-japanischen Krieges im Jahre 1937 und die Problematik der Modernisierung bis hin zu Fragen der ökonomischen Entwicklung der Volksrepublik und zur Kulturrevolution.

Der zweite, von Ping-ti Ho herausgegebene Band ist der Außenpolitik gewidmet. Die Autoren dieses Bandes sind Richard Löwenthal, Uri Ra'anan, Norton Ginsburg, Hans Morgenthau, Robert Scalapino, Morton Halperin, Frank Armbruster, Harold Hinton, Donald Zagoria, Roger Hilsman, Davis Bobrow, David Mozingo, Ruth McVey, Wayne Wilcox und A. M. Halpern. Die Merkmale der chinesischen Außenpolitik, die "Zwei China"-Frage, das Problem der Atomwaffen sowie das Verhältnis Chinas zu den USA, zu Vietnam, zu Indonesien und zu Japan sind Themen der Beiträge, die wiederum von einer Reihe anderer Autoren kommentiert werden. Es ist nicht möglich, in dieser Rezension auf alle diese Beiträge einzugehen. Deshalb soll vertretend für alle aus jedem der beiden Bände ein Beitrag ausführlicher vorgestellt werden:

Ping-ti Ho: Salient Aspects of China's Heritage (Band I, S. 1 ff.) und Hans J. Morgenthau: The United States and China (Band II, S. 93 ff.). Diese Auswahl wurde von der Überlegung geleitet, daß in der öffentlichen Diskussion China allzuoft isoliert von seiner historischen Entwicklung gesehen wird und daß heute das chinesisch-amerikanische Verhältnis zu einer der entscheidenden internationalen Beziehungen werden kann.
Für Ping-ti Ho führt gewissermaßen die ganze chinesische Geschichte auf das heutige Staatswesen zu. Denn erst die dem modernen Staat zur Verfügung stehenden Kontrollmittel haben das immer wieder erstrebte "Groß-China“ zu einer unumstößlichen Tatsache werden lassen; das Ziel der chinesischen Geschichte ist damit erreicht (S. 7).

Ho stellt drei Merkmale heraus, die sich durch die chinesische Geschichte hinziehen und aufgrund derer man auch die Erscheinungen in der heutigen Volksrepublik nicht als umwälzend bezeichnen kann:

1. Jede Dynastie in der chinesischen Geschichte von 221 v. Chr. (Ch'inDynastie) bis zur Volksrepublik (seit 1949) gründete sich ohne Ausnahme auf militärische Macht (S. 16).

2. Die gesamte chinesische Geschichte war durch ihren Herrscher-Kult gekennzeichnet. Weil eine Herrscherfigur im alten Sinne fehlte, war die frühe republikanische Periode Chinas nach 1911 eine der chaotischsten der chinesischen Geschichte. Und der Grund dafür, daß die Volksrepublik China der Entstalinisierung der Sowjetunion nicht folgt, ist nicht darin zu erblicken, daß China etwa politisch, ökonomisch, sozial oder ideologisch mit der Sowjetunion in den frühen Stalinjahren zu vergleichen ist, sondern weil in China seit mehr als 2000 Jahren ein Regierungssystem undenkbar ist, das nicht mit einer charismatischen Führerpersönlichkeit identifiziert werden könnte (S. 16-18).

3. Die absolutistische Herrschaft in China war niemals irgendwelchen institutionellen Kontrollen unterworfen. Die Herrscher hatten immer die Möglichkeit, über die Regierung hinwegzuregieren, und noch in der Periode der Nationalregierung lag die Schaffung verschiedener geheimer Behörden auf dieser historischen Linie (S. 18-25).

Für Ho lagert daher der "neue Wein“ der maoistischen Ideologie und der beispiellosen Macht der Organisation nur in den „2000 Jahre alten Schläuchen “ des autoritären Staates (S. 25).

Auch für den Konfuzianismus, der die Geschichte Chinas geformt hat und der 
dem Menschen die Fähigkeit zuschreibt, seine Umwelt und damit den menschlichen Charakter zu formen und zu verbessern, sieht der Autor eine Parallele im unerschütterlichen Glauben der chinesischen Kommunisten an die Allmacht des Menschen (S. 30 f.).

Die Frage, ob die Volksrepublik mit allen ihren Außerungen und Reaktionen nur eine Fortsetzung der chinesischen Geschichte oder aber eine besonders gefährliche Spielart der Anwendung kommunistischer Ideologie darstellt, ist eine ungelöste Frage der Chinakunde. Herbert Franke sieht denn auch die Gefahr, daß das Heimweh nach der Vergangenheit die Tendenz nährt, die Auswirkungen des Kommunismus zu verharmlosen (S. $50 \mathrm{FN}$ ). Er warnt vor einer zu weitgehenden Parallelisierung: Wir können zwar etwas über das Leben des Volkes im heutigen China aussagen; aber wir besitzen kein einziges Dokument über das Leben des Volkes im alten China, das nicht durch die literarische Tradition verbrämt und möglicherweise verfälscht ist.

Es wäre jedoch sicher auch falsch, die Volksrepublik isoliert von der chinesischen Geschichte zu sehen - und wenn es dem Laien nur dazu dient, festzustellen, warum das chinesische Volk wie selten eines so geschichtsbewußt und stolz auf seine Größe ist.

Der zweite hier ausgewählte Beitrag über die chinesisch-amerikanischen Beziehungen hat ein nicht minder umstrittenes und sehr aktuelles Thema zum Inhalt: die chinesisch-amerikanischen Beziehungen. Auch Morgenthau geht von der chinesischen Geschichte aus. China hat sich immer als das Zentrum des politischen Universums gesehen, für das es nicht nötig ist, sich um ein Verständnis der Außenwelt $\mathrm{zu}$ bemühen (S. 94). Es scheint daher auch nicht verwunderlich zu sein, daß auch im heutigen China das elementarste Verständnis für die Außenwelt fehlt (S. 95). In einer zusammenwachsenden Welt kann jedoch erwartet werden, daß die chinesischen Führer aus der Erfahrung lernen und ihre Politik den tatsächlichen Gegebenheiten anpassen werden.
Auf amerikanischer Seite ist Ausgangspunkt der Betrachtung die „Politik der offenen Tür", die anfangs dazu diente, den freien Wettbewerb um die Ausbeutung Chinas zu gewährleisten. Bald jedoch ging es den USA um die Erhaltung des politischen Gleichgewichts in Asien, das gestört worden wäre, wenn sich irgendeine europäische oder auch asiatische Macht mit dem gewaltigen Potential Chinas verbunden hätte (S. 97). Dieses Gleichgewicht mußte durch Stärkung Chinas erhalten werden. China wurde damit zum Eckpfeiler dieser Gleichgewichtspolitik, bis die Kommunistische Partei die Regierungsgewalt übernahm. Seitdem verfolgten die USA eine Politik der Isolierung und der Eindämmung.

Die Politik der Isolierung ist ohne jeden Erfolg geblieben, so daß auch nicht erwartet werden kann, daß ihre Aufgabe von China honoriert werden würde (S. 99). Die Politik der militärischen Eindämmung geht von der falschen Annahme aus, daß eine militärische Bedrohung vorliegt, der wie in Europa tatsächlich mit militärischen Mitteln begegnet werden könnte. Die Bedrohung, die von China ausgeht, ist jedoch politischer Art und wirkt sich in erster Linie im Falle schwacher Regierungen und $\mathrm{Ge}-$ sellschaften aus. In solchen Fällen kann die militärische Eindämmung sogar eher das Gegenteil dessen bewirken, was sie beabsichtigt (S. 100 f.).

Morgenthau ist der Ansicht, daß die von den USA praktizierten Nadelstiche an der Peripherie Chinas wirkungslos bleiben. Die Macht im Inneren Chinas müßte konsequenterweise zerstört werden. China als unterentwickeltes Agrarland kann aber nur überwunden werden, wenn es erobert wird (S. 101), was nicht möglich ist. Auch die nukleare Drohung der USA schreckt China so lange nicht, wie es unterentwickelt bleibt.

Die USA müssen sich nach Meinung des Autors mit der Situation abfinden und die Politik der militärischen Eindämmung liquidieren. $\mathrm{Da}$ die chinesische Expansion politischer Natur ist, kann ihr nur politisch begegnet werden, indem 
man nämlich die Völker Asiens politisch, sozial und ökonomisch stärkt und nicht versucht, sie gleichzeitig in gegen China gerichtete politische oder militärische Allianzen zu zwingen (S. 104). Die Politik der militärischen Eindämmung muß nach Morgenthau früher oder später zum Krieg mit China führen. Für die USA gibt es nur die Alternative, „entweder die Mittel, die wir anzuwenden bereit sind, unseren Zielen anzupassen, oder unsere Ziele auf das Niveau der Mittel herabzusetzen, die wir anwenden wollen" (S. 107).

Sollte die Politik der militärischen Eindämmung fortbestehen, so gibt es nach Morgenthau offenbar nur einen Trost, der dem "Gleichgewicht des Sckreckens" nahekommt; Chinas Nuklearmacht wird wachsen, damit aber auch verletzlicher werden. Wachsende Nuklearmacht und steigende Verletzlichkeit eines Landes gehen Hand in Hand und veranlassen die Politik zu vorsichtigerem Taktieren (S. 103). Ob das jedoch einem Sieg der Vernunft gleichzusetzen ist, darf füglich bezweifelt werden.

Morgenthau findet im Kommentar Paul A. Vargs eine Kritik, die dem Leser einen Hauch von der Diskussion vermittelt, die diese Konferenz der Universität Chicago beherrscht haben muß und deren Beiträge nicht nur wertvolle Informationen. zum Verständnis des heutigen China, sondern auch in reichem Maße Stoff zum Nachdenken für den am Problem China interessierten Bürger aller der Staaten geliefert hat, für die das Verhältnis zu China noch der klaren Stellungnahme harrt.

Bernhard Großmann

James C. Scott

Political Ideology in Malaysia

Reality and the Beliefs of an Elite,

New Haven and London, Yale

University Press 1968, X + 302 S.

Das Programm, das der Verfasser sich gesetzt hat, ist ehrgeizig und vielversprechend: Er will nicht nur die politische Ideologie Malaysias als eines im Übergang zur Modernisierung befindlichen Landes erfassen, die Ergebnisse dieses Bemühens sollen vielmehr überdies paradigmatische Bedeutung für andere neue, sich ebenfalls modernisierende Staaten haben. Die hochgespannten Erwartungen werden jedoch arg enttäuscht; es ist dem Verfasser nicht gelungen, das aus der Situation des Uberganges zur Modernität resultierende Spezifische der malaysischen Ideologie herauszuarbeiten, und erst recht ist es ihm nicht geglückt, das für andere sich modernisierende Staaten Typische der für Malaysia gewonnenen Erkenntnisse darzutun. Zwar fehlt es nicht an Versuchen in letzter Hinsicht (vgl. etwa $101,103,137,192,210 / 212$ ), aber sie wirken durchaus krampfhaft und überzeugen nicht.

Befragung von regelmäßig anderthalb Dutzend ausgewählter Bürokraten ist der Weg, den der Verfasser für die Erforschung der Ideologie Malaysias gewählt hat. Aber die Antworten der Befragten lassen so gut wie nichts Spezifisches erkennen: Welcher Ministerialbeamter z. B. irgendwo und irgendwann hätte sich niemals über seinen politischen Minister geärgert? (234)

Entsprechend verhält es sich mit den Ergebnissen, die der Verf. vorführt. Besonders stolz scheint er auf die Entdekkung einer Denkweise zu sein, die er durch das Bild vom "constant pie“ zu bestimmen sucht. Gemeint ist hiermit die Úberzeugung einer Gruppe, daß das verfügbare Sozialprodukt unveränderlich feststehe, und daß es ihren Mitgliedern somit nur noch darauf ankommen könne, sich einen möglichst großen Anteil an dem gleichbleibenden Kuchen zu sichern. Die Zuordnung eines solchen Denkens zu Nationen, die sich zu Modernisierung entschlossen haben, scheint mir geradezu falsch; denn sinnvollerweise setzt ein solcher Entschluß voraus, daß man von der Möglichkeit einer Steigerung des Sozialproduktes überzeugt ist und von dieser Möglichkeit energischen Gebrauch zu machen gedenkt.

Nach alledem ist es nicht gestattet, in dem Buch einen förderlichen Beitrag zur Durchdringung der Probleme zu sehen, die sich die neuen Staaten mit dem Entschluß zur Modernisierung gestellt haben.
Herbert Krüger 